\title{
Dynamical geometry for multiscale dissipative particle dynamics
}

\author{
G. De Fabritiis ${ }^{\mathrm{a}}$, P. V. Coveney ${ }^{\mathrm{b}}$ \\ ${ }^{a}$ Centre for Computational Science, Queen Mary, University of London, \\ Mile End Road, London E1 $4 N S$, UK \\ ${ }^{\mathrm{b}}$ Centre for Computational Science, Department of Chemistry, \\ University College London, 20 Gordon Street, London WC1H OAJ
}

\begin{abstract}
In this paper, we review the computational aspects of a multiscale dissipative particle dynamics model for complex fluid simulations based on the feature-rich geometry of the Voronoi tessellation. The geometrical features of the model are critical since the mesh is directly connected to the physics by the interpretation of the Voronoi volumes of the tessellation as coarse-grained fluid clusters. The Voronoi tessellation is maintained dynamically in time to model the fluid in the Lagrangian frame of reference, including imposition of periodic boundary conditions. Several algorithms to construct and maintain the periodic Voronoi tessellations are reviewed in two and three spatial dimensions and their parallel performance discussed. The insertion of polymers and colloidal particles in the fluctuating hydrodynamic solvent is described using surface boundaries.
\end{abstract}

\section{Introduction}

The non-equilibrium behaviour of complex fluids continues to present a major challenge for both theory and numerical simulation. Such fluids include multiphase flows, particulate and colloidal suspensions, polymers, amphiphilic fluids, including emulsions and microemulsions, and other fluids where Brownian motion is important. Over the last decade several strategies have been introduced both from a microdynamical point of view and from a continuum or macro-dynamical point of view. This article is particularly focused on mesoscopic/particulate approaches, but the Voronoi tessellation has been employed for similar applications to model colloidal particles and polymers by solving conventional Navier-Stokes like equations with moving boundary conditions (see [1] and references therein).

Preprint submitted to Elsevier Science $\quad 7$ September 2018 
A recent contribution to the family of bottom-up approaches is the dissipative particle dynamics (DPD) method introduced by Hoogerbrugge and Koelman in 1992 [2]. Successful applications of the technique have been made to colloidal suspensions [3], polymer solutions [4] and binary immiscible fluids [5]. Dissipative particle dynamics has been shown to produce the correct macroscopic (continuum) theory; that is, for a one-component DPD fluid, the Navier-Stokes equations emerge in the large scale limit, and the fluid viscosity can be computed [6,7]. However, even though dissipative particles have generally been viewed as clusters of molecules, the cluster of molecules are not considered as a thermodynamic system itself. In a following development of the DPD model, Flekkøy and Coveney [8,9,10] and Serrano and Español [11] derived a new model which provides a precise definition of the term 'mesoscale' and enables us to retrieve numerically the specific thermodynamics of the solvent in the sense that the exact Gibbs equilibrium is approached, the entropy is an increasing function in time, and the total energy is exactly conserved [12]. In this approach, the fluid dissipative particles are defined as cells on a Voronoi lattice with variable sizes and masses; the Voronoi tessellation must be updated at each time step under the dynamics of the model. The scheme enables one to select any desired local scale for the mesoscopic description of a given problem. Indeed, the method may be used to deal with situations in which several different length scales are simultaneously present.

In this article, we discuss the computational aspects of the model and its computational overhead due to the Voronoi tessellation; in particular, we present the development of an efficient $\mathrm{C}++$ code for the implementation of the new fluid particle model. Our discussion concentrates on the challenging aspects of maintaining the Voronoi tessellation over time, with various kinds of boundary conditions.

\section{Multiscale dissipative particle dynamics}

In our case, we follow the model described in detail in $[11,12]$, but we apply its isothermal version, which has the advantage of greater simplicity. In fact, if heat diffusion is not important for the system under consideration, then the isothermal model furnishes an efficient alternative, because several simplifications are possible in the equations (for instance, the Itô-Stratonovich terms all vanish). Defining the state of a fluid particle by its position, mass and momentum $\mathbf{x}=\left\{\mathbf{r}_{i}, M_{i}, \mathbf{P}_{i}\right\}$, the resulting equations for the isothermal model are

$$
d \mathbf{r}_{i}=\mathbf{v}_{i} d t
$$




$$
\begin{aligned}
d M_{i} & =\sum_{j} \frac{\rho_{i}+\rho_{j}}{2} \mathbf{C}_{i j} \cdot \mathbf{v}_{i j} d t \\
d \mathbf{P}_{i} & =\sum_{j} \frac{A_{i j}}{2}\left(p_{j i} \mathbf{1}+\boldsymbol{\Pi}_{i}+\Pi_{i} \mathbf{1}\right) \cdot \mathbf{e}_{i j} d t+d \widetilde{\mathbf{P}}_{i} \\
& +\sum_{j} \frac{\rho_{i}+\rho_{j}}{2} \frac{\mathbf{v}_{i}+\mathbf{v}_{j}}{2} \mathbf{C}_{i j} \cdot \mathbf{v}_{i j} d t .
\end{aligned}
$$

where $\mathbf{v}_{i}=\mathbf{P}_{i} / M_{i}$ is the velocity, $\rho_{i}=M_{i} / V_{i}$ is the mass density, and $d$ is the spatial dimension. For a general variable $x$, we write $x_{i j}=x_{i}-x_{j}$. The pressure $p_{i}$ is given through the equilibrium equations of state once the temperature $T$ is assigned. We have also introduced geometric quantities arising from the Voronoi construction: $A_{i j}$ is the area (length in 2D) of the face between cells $(i, j), \mathbf{e}_{i j}=\left(\mathbf{r}_{i}-\mathbf{r}_{j}\right) / r_{i j}$ with $r_{i j}=\left|\mathbf{r}_{i}-\mathbf{r}_{j}\right|$ is the unit vector normal to the face $(i, j)$ and, finally, $\mathbf{C}_{i j}=\frac{A_{i j}}{r_{i j}}\left(\mathbf{A}_{c m}-\frac{\mathbf{r}_{i}+\mathbf{r}_{j}}{2}\right)$ is a geometrical vector parallel to the face $(i, j)$. The dissipative stress tensor is

$$
\boldsymbol{\Pi}_{i}^{\alpha \beta}=\frac{\eta_{i}}{V_{i}}\left[\frac{1}{2} \sum_{j} A_{i j}\left[\mathbf{e}_{i j}^{\alpha} \mathbf{v}_{j}^{\beta}+\mathbf{e}_{i j}^{\beta} \mathbf{v}_{j}^{\alpha}\right]-\frac{1}{D} \delta^{\alpha \beta} \sum_{j} A_{i j} \mathbf{e}_{i j} \cdot \mathbf{v}_{j}\right]
$$

while the fluctuations in momentum are

$$
d \widetilde{\mathbf{P}}_{i}=\sum_{j} \frac{1}{2} A_{i j} \mathbf{d} \widetilde{\sigma}_{j} \cdot \mathbf{e}_{i j}
$$

where the stress tensor is

$$
\mathbf{d} \widetilde{\sigma}_{i}=\left(4 k_{B} T_{i} \frac{\eta_{i}}{V_{i}}\right)^{\frac{1}{2}} d \overline{\mathbf{W}}_{i}^{S}+\left(2 D k_{B} T_{i} \frac{\xi_{i}}{V_{i}}\right)^{\frac{1}{2}} \operatorname{tr}\left[d \mathbf{W}_{i}\right]
$$

Here, $d \mathbf{W}_{i}$ is a matrix $(d \times d)$ of independent Wiener increments, i.e. $d W_{i}^{(\alpha, \beta)}$ has zero mean and variance $d t$ and is uncorrelated to any other random variables, $d \overline{\mathbf{W}}_{i}^{S}=\frac{1}{2}\left(d \mathbf{W}_{i}+d \mathbf{W}_{i}^{t}\right)-\frac{1}{d} \operatorname{tr}\left[d \mathbf{W}_{i}\right] \mathbf{1}$ is a symmetric traceless matrix.

The SDEs (1) can be integrated numerically using any stochastic integrator, such as Euler, Heun or higher order schemes [13]. For our simulations, we used an integrator based on a generalisation of the Trotter formula to the stochastic case [14] which proved to be simple to apply and very efficient. 


\section{Voronoi tessellation}

The model is centrally based on the Voronoi tessellation. The Voronoi tessellation is simply defined by a set of points $P=\left\{p_{1}, \ldots, p_{N}\right\}$ in Euclidean space; a partition of the space assigning every point to its nearest site is called a Voronoi tessellation. The Voronoi cell $V\left(p_{i}\right)$ consists of all the points at least as close to $p_{i}$ as to any other site

$$
V\left(p_{i}\right)=\left\{x:\left|p_{i}-x\right| \leq\left|p_{j}-x\right|, \forall j \neq i\right\}
$$

The Voronoi tessellation can be computed through the Delaunay triangulation, which is the triangulation that maximises the minimum internal angle among the triangles [15]. In terms of the fluid dissipative particles, the Delaunay triangulation indicates the neighbourhood of each fluid particle in the tessellation and the Voronoi tessellation indicates its spatial extension (see Figure 2). By first triangulating the set of points $P$ with the Delaunay triangulation, the Voronoi tessellation can then be constructed by using the Delaunay-Voronoi duality [15], i.e. to each Delaunay vertex corresponds a Voronoi region and to each Delaunay edge corresponds a Voronoi boundary surface or line in two dimensions.

The physical model requires maintenance of the Voronoi tessellation for each time step following the dynamics in the Lagrangian frame of reference and specification of the boundary conditions for the tessellation. In our opinion, direct coding of the construction of the Voronoi tessellation is not desirable. This still is in its own right a research field in computational geometry in order to obtain a stable and efficient triangulation. It involves writing exact number types to check the validity of predicates on points (for instance the insphere property for four points) and combinatorial aspects to handle the data structure. Nevertheless, writing an inefficient and unstable code to construct the Voronoi tessellation is quite simple, but the result will likely be orders of magnitude slower and unstable in most practical situations. Whilst there are several public libraries $[16,17]$ that are able to compute the Voronoi-Delaunay tessellation for a given set of points. However, the algorithmic complexity of the construction is at best $N \log N$, where $N$ is the number of points, and thus it is crucial to use an efficient library to match that complexity. We were unable to find any library able to dynamically change the tessellation and impose periodic boundary conditions.

Previously, in our research group, the Triangle library [16] was employed for the construction of the Voronoi tessellation [18]. Although the Triangle library does not support three dimensional Voronoi tessellations, it is the fastest library known to us in two dimensions. Triangle is based on the divide-andconquer algorithm [15], which allows one to compute the tessellation given the 
complete list of points. It furnishes as well an incremental algorithm, which provides the functions to construct the tessellation point by point.

With Triangle the Voronoi tessellation was maintained over time by deleting the set of points $P$ from the tessellation, determining the new positions of the points and then computing the tessellation for the new points, for every time-step. Given the algorithmic complexity of the construction of Voronoi tessellations, this is clearly not an efficient way to maintain the tessellation over time; it is desirable to be able to locally restore the tessellation at each time-step, thus avoiding deleting and then re-computing the entire tessellation.

Subsequent awareness of the Computational Geometry Algorithms Library (CGAL) [17] gave us a homogeneous environment for two and three dimensional tessellations. CGAL incorporates a wide-range of function modifiers, including the insertion or removal of specific points in a tessellation, thus offering a highly flexible code for the construction and maintenance of a Voronoi tessellation over time. Consequently, we are currently employing the CGAL library for the development of the Voronoi DPD code in two and three spatial dimensions.

\section{Boundary conditions}

In this section, we describe possible implementations of periodic and wall boundary conditions in two and three dimensions based on the insertionremoval of image cells (points) around the simulation box. The insertion of polymers and colloidal particles is described using boundary surfaces. We present these algorithms in two dimensions because our three dimensional implementation is a straightforward application of the same techniques. The library takes care of the extra complexity of handling the three dimensional tessellation. Differences are pointed out in Section 4.4.

\subsection{Periodic boundary conditions}

Boundary conditions can be implemented by inserting 'image points' around the boundaries of the space domain. We call image points the points of the tessellation which lie outside the simulation box and represent images of centres of fluid particles inside the simulation box. The simplest way to achieve a periodic tessellation is to copy the simulation region to the eight surrounding boundary regions (see Figure 3).

However, this requires that the tessellation has to be computed for a total of 


\section{Algorithm 1 (PERIODIC BOUNDARY CONDITIONS)}

(1) Insert the corner points of the simulation domain

(2) Triangulate (Move) $N$ points

(3) for every finite Delaunay triangle $\left(p_{1}, p_{2}, p_{3}\right)$

\{

Compute the circumcircle $C(V)$

if $C(v)$ intersects a boundary face(s)

\{

Copy $p_{1}, p_{2}$ and $p_{3}$ to the boundary region(s)

of the opposite face(s)

\}

\}

(4) Remove the corner points

$9 N$ points if $N$ is the number of points to be triangulated in the simulation region. It is obviously not necessary to triangulate this number of image points to maintain the periodic boundary conditions and in light of the algorithmic complexity of the construction of the tessellation, it is crucial to minimise the number of image points. Clearly, it is only the points that lie 'near' a boundary face that must be copied to the boundary regions. To isolate these 'boundary points' we use the following geometrical criterion: a point is in the neighbourhood of the boundary if the presence of another point outside the domain can affect its Voronoi region. This criterion can be implemented with a simple algorithm. This is given in Algorithm 1.

In the first step of the algorithm, $N$ points are triangulated in the simulation region. In step 2, the following geometrical properties of the Delaunay-Voronoi tessellation [15] are employed:

- Each node $p_{i}$ of the Delaunay triangulation corresponds to a Voronoi region $V\left(p_{i}\right)$.

- If $v$ is a Voronoi vertex at the junction of $V\left(p_{1}\right), V\left(p_{2}\right), V\left(p_{3}\right)$ then $v$ is the centre of the circle $C(v)$ determined by the points $p_{1}, p_{2}$ and $p_{3}$.

- $C(v)$ is the circumcircle for the Delaunay triangle $\left(p_{1}, p_{2}, p_{3}\right)$ containing no other points $p_{i}$.

It follows from the Delaunay-Voronoi properties above that for a Delaunay triangle $\left(p_{1}, p_{2}, p_{3}\right)$, if the circumcircle $C(v)$ does not intersect a boundary face of the domain, then no image point can affect the Voronoi regions $V\left(p_{1}\right), V\left(p_{2}\right)$ and $V\left(p_{3}\right)$ and hence, according to our geometrical criterion, $p_{1}, p_{2}$ and $p_{3}$ do not have to be copied to the boundary regions. However, if $C(v)$ does intersect a boundary face, then an image point can affect the Voronoi regions $V\left(p_{1}\right)$, 
$V\left(p_{2}\right)$ and $V\left(p_{3}\right)$. Unfortunately, it is not possible to know if any boundary points will produce an image point within $C(v)$ until the tessellation for these boundary points is constructed. Nevertheless, a good upper-bound can be given on the number of points that must be copied by considering the worst situation that for any Delaunay triangle $\left(p_{1}, p_{2}, p_{3}\right)$ whose circumcircle $C(v)$ interests a boundary face, an image point will always be located within $C(v)$. In this case $p_{1}, p_{2}$ and $p_{3}$ must be copied to the boundary region of the opposite face; if $C(v)$ intersects the corner (i.e. two boundary faces), $p_{1}, p_{2}$ and $p_{3}$ must be copied to the boundary regions of the two opposite faces and also to the boundary region of the opposite corner (see Figure 3).

The points which belong to the convex hull of the triangulation have their Voronoi centre $v$ positioned at infinity and thus it is not possible to compute $C(v)$ in these cases. The solution we applied is to insert the corner points of the periodic boundary regions in order to create an external convex hull. After the image points are calculated the corners points are removed. This is not needed when the periodic boundary condition is already set and we want to maintain it, because the image points will form the convex hull.

The periodic boundary tessellation produced by Algorithm 1 is shown in Figure 4. Thus Algorithm 1 avoids copying all the points and, in assuming the worst case scenario that an image point is always located within $C(v)$ for any boundary point, this algorithm reduces the number of image points to the minimum upper-bound possible.

\subsection{Fixed (solid) wall boundary conditions}

A possible way to implement fixed (solid) wall boundary conditions for the Voronoi tessellation is to introduce image points into the boundary regions which correspond to the reflection of the boundary points in their boundary face(s) (see Figure 5). The interaction between a boundary particle and the 'wall' is then handled by the interaction between the particle and its 'mirrorimage' particle. We have successfully implemented fixed wall boundary conditions for the Voronoi tessellation with Algorithm 2.

The algorithm follows the same principle as Algorithm 1; we first insert the corner points of the simulation domain, and then triangulate our $N$ points, therefore enabling us to compute the circumcircle of every Delaunay triangle in the simulation domain. Next in step 3, we isolate all of the boundary points. However, the criterion for a boundary point for fixed wall boundary conditions is more complicated than that for periodic boundary conditions. For any circumcircle $C(V)$ which intersects a boundary face, two additional requirements must be satisfied before $p_{1}, p_{2}$ or $p_{3}$ can be isolated as boundary 


\section{Algorithm 2 (FIXED WALL BOUNDARY CONDITIONS)}

(1) Insert the corner points of the simulation domain

(2) Triangulate $N$ points.

(3) for every finite Delaunay triangle $\left(p_{1}, p_{2}, p_{3}\right)$

\{

Compute the circumcircle $C(V)$

if $\left[C(v)\right.$ intersects a boundary face AND $\left(p_{1}, p_{2}, p_{3}\right)$ lies on the correct side of the boundary]

\{

for each Delaunay vertex, if the perpendicular projection of the vertex on to the supporting line is also on the boundary face segment, reflect the vertex in the boundary face

\} \}

(4) Remove the corner points

points.

Firstly, for a given Delaunay triangle $\left(p_{1}, p_{2}, p_{3}\right)$, if $C(V)$ intersects a boundary face, it is possible for $\left(p_{1}, p_{2}, p_{3}\right)$ to lie on the wrong side of the boundary. For example, consider the fixed wall boundary conditions for the ' $\mathrm{C}$ ' shaped domain in Figure 5. Suppose we are isolating the particles that lie in the neighbourhood of the first 'shorter' horizontal boundary face from the bottom in Figure 5. It is possible for the circumcircle of a Delaunay triangle $\left(p_{1}, p_{2}, p_{3}\right)$ lying above this boundary face to intersect this boundary and yet clearly $\left(p_{1}, p_{2}, p_{3}\right)$ cannot physically lie in the neighbourhood of this boundary. Thus, if $C(V)$ intersects a boundary face, we must then check that $p_{1}, p_{2}$ and $p_{3}$ lie on the correct side of the boundary; if we are traversing the boundary faces in a clockwise direction then $p_{1}, p_{2}$ and $p_{3}$ are on the correct side of the boundary face if they lie to the right-hand side of the boundary. Similarly, an anti-clockwise traversal of the boundary faces requires that $p_{1}, p_{2}$ and $p_{3}$ lie to the left-hand side of the boundary.

If these first two requirements are met, then we must check one final geometrical property. We refer again to the first 'shorter' horizontal boundary face up from the bottom of the ' $\mathrm{C}$ ' shaped domain in Figure 5. Suppose for a Delaunay triangle $\left(p_{1}, p_{2}, p_{3}\right)$, the circumcircle $C(V)$ intersects this boundary face and $p_{1}, p_{2}$ and $p_{3}$ lie on the correct side of the boundary. Consider the supporting line of this boundary face (that is, the infinite line which is parallel to the boundary face segment and passes through it) and the perpendicular projection of the Delaunay vertices $p_{1}, p_{2}$ and $p_{3}$ onto this supporting line. For the majority of cases, these three projections will also lie on the boundary face 
segment in which case $p_{1}, p_{2}$ and $p_{3}$ can be reflected in the boundary face and are thus boundary points. However, for Delaunay triangles whose circumcircle intersects the left-end of this boundary face, it is possible that some or even all of these projections may not lie on the boundary face segment, that is, they do not have a reflection in this boundary face and hence are not boundary points. Thus, if $C(V)$ intersects a boundary face and $p_{1}, p_{2}$ and $p_{3}$ lie on the correct side of the boundary, we must then determine if the perpendicular projection of each Delaunay vertex onto the supporting line is also on the boundary face segment. For those that are, they are boundary points.

Having isolated the boundary points with the above criterion, we then insert the reflection of these points in their boundary face(s). We note that if $C(V)$ intersects a corner (i.e. two boundary faces) then the two additional geometrical requirements detailed above must be applied separately to each boundary. Finally in step 4 of Algorithm 2, we remove the corner points from the simulation.

This algorithm can be applied to any two dimensional polygon that can be described by a set of vectors. We show in Figure 5 the fixed wall boundary tessellation for a ' $\mathrm{C}$ ' shaped domain, obtained using Algorithm 2. We note that for corners where the angle between the two boundary faces in the fluid is greater than $180^{\circ}$, the actual corner is not produced exactly. This is because near the corner, the insertion of an image point above one of the boundary faces will affect the Voronoi region of the other boundary face (see Figure 5). However, for realistic simulations involving tens of thousands of points, the 'approximate' corner obtained is perfectly sufficient.

\subsection{Boundary surfaces}

One final aim of this research is to add specificity to the model by inserting polymers and colloidal particles in the fluctuating hydrodynamic solvent based on the Voronoi dissipative particle dynamics model. Then, the rheological properties of the resulting system can be computed via direct simulations of shear flows.

Polymers and colloidal particles are inserted in the Voronoi tessellation using surface boundaries formed by chains of Voronoi centres. This is represented in Figures 6 and 7. The boundary surface are fixed giving a couple of Voronoi centres sufficiently close to form a Voronoi surface, which represents the boundary. How close they need to be is computed from the typical scale of the system or the smallest Voronoi fluid particle.

The physical meaning of the two Voronoi centres depends on their position. The Voronoi cell internal to the polymer or the colloid is a coarse-grained 
representation of a part of the polymer. The Voronoi centre in the fluid is a fluid particle which does not slip on the colloidal/polymer surface.

This description of surface boundary could be used as well for wall boundaries, with the disadvantage that an higher number of cells is needed to construct the boundary compared to the approach presented in Section 4.2, but with the advantage of an easy construction and maintenance of the boundary during the dynamics. In fact, once the two cells forming the boundary surface are kept at a fixed distance, then the maintenance of the tessellation does not involve any particular extra work.

\subsection{Efficiency of image point boundary conditions}

The efficiency of the construction of periodic/solid boundary conditions using image points depends on the number of images that we need to insert. Intuitively, this is a limitation when we tessellate a small number of points, because in this case the surface:volume ratio is high. We quantitatively measured the number of images needed to impose boundary conditions in two and three spatial dimensions.

The results are reported in Table 1 . First, we note that while in two dimensions we have 8 neighbour regions around the box, in three dimensions there are 26 . The effect of this geometrical change is evident. The number of shell points

represents the number of points that have at least one image. The number of fluid particles which are in the core, and therefore not affected by boundary conditions, is of course $N=N_{f p}-N_{\text {shell }}$, where $N_{f p}$ is the number of fluid particles. Each point in the shell could be copied to more than one neighbour region. The number of images is the number of points that are actually inserted outside the simulation box. The number of extra images gives the number of point images that are not connected to any point inside the simulation domain, i.e. their presence does not affect the periodic boundary at all. The small number of extra images compared to the number of fluid particles shows that the criterion used to select image points is very effective.

Obviously, it is important that the number of images, which represent a significant computational overhead, is small compared to the number of points in the box. This is easily obtained in two dimensions where for 1000 points we have 190 images (less than 20\%). However, in three dimensions, to have the same percentage we need more than 50000 points. This severely limits the performance of the current code with full periodic boundary conditions in three dimensions. 


\section{Maintaining the Voronoi tessellation dynamically}

The dynamical maintenance of the tessellation is based on the reconnection algorithm (see [19]). The reconnection algorithm locally restores the Delaunay property on a valid triangulation. It is very effective, but we need to guarantee that after all the points are moved the tessellation does not present invalid triangles (tetrahedra). It is not simple to assure this condition. Usually, by reducing the time step it is possible to control the validity of the triangulation. However, in our opinion this method is not really stable, and brings with it the additional problem that the reduction of the time step effectively slows down the code. In three dimensions, we prefer to locally restore the tessellation moving one point by one. We are thus able to check that the triangulation is still valid and then to use asynchronous dynamics to update different fluid particles with different time steps. This last option is particulary interesting for a multiscale model like this. In Appendix A, we detail the code for the maintenance of the tessellation in two and three dimensions realised with the CGAL library [17].

\subsection{Local reconnection algorithm in two dimensions}

Given the algorithmic complexity of the construction of a Voronoi tessellation for $N$ points $(N \log N)$, it is crucial that we are able to efficiently maintain the Voronoi tessellation over time. We can make significant improvements by utilising the flexibility of the CGAL library. We are able to restore the Delaunay Triangulation (and thus the Voronoi tessellation) locally with the following algorithm (an implementation for the CGAL library is shown in Appendix A), which is an extension of the algorithm used by CGAL for restoring the Delaunay triangulation when individual points are inserted or removed.

We now consider the individual steps of the algorithm. In the first step we ensure that no point can move outside its Voronoi region by restricting the size of the time-step. This is achieved by iterating over the edges of the Delaunay triangles and computing the time-step for each edge such that neither point can move more than a quarter the distance towards the other one by setting $\delta t$ to the minimum of all these values. After integrating the stochastic Langevin equations of motion, we then update the position of each point in step 2. It is clear that some triangles may now be invalid Delaunay triangles and so we restore the triangulation to a Delaunay triangulation in step 3. We achieve this as follows: every triangle in the simulation domain has three neighbouring triangles, that is, three neighbouring vertices (by definition, the triangles that belong to the convex hull have the infinite vertex as one of their three neighbouring vertices). A triangle is a valid Delaunay triangle if it sat- 


\section{Algorithm 3 (RESTORE DELAUNAY)}

(1) Compute $\delta t$ such that no point can move outside its Voronoi region

(2) Move the points

(3) for every finite Delaunay triangle $\left(p_{1}, p_{2}, p_{3}\right)$

\{

Compute the circumcircle $C(v)$

if $C(v)$ does not satisfy the empty circle property

\{

Triangulate the quadrilateral formed by $p_{1}, p_{2}, p_{3}$ and the point lying within $C(v)$ the other way round

\}

isfies the empty circle property, that is, if none of its neighbouring vertices lies inside its circumcircle. Therefore, we iterate over the finite triangles in the triangulation and check the empty circle property for every triangle; for any triangle $\left(p_{1}, p_{2}, p_{3}\right)$ which does not satisfy the empty circle property due to a neighbouring vertex, say $q$, located within its circumcircle, we simply triangulate the quadrilateral formed by $p_{1}, p_{2}, p_{3}$ and $q$ the other way round. We note that Algorithm 3 must be implemented such that any new triangles formed are subsequently visited by the iteration process.

Clearly, there is far less work involved in restoring the Delaunay triangulation than with re-computing the whole tessellation for every time-step. Indeed, the above restore Delaunay algorithm is a very efficient way of maintaining a two-dimensional Voronoi tessellation over time.

\subsection{Reconnection algorithm in three dimensions}

In three dimensions, instead of performing a sequence of geometrical flips of the facets in order to locally restore the Delaunay property, we check the validity of the insphere property for all the facets and eventually, if the test fails, we move back the point to the preceding position, remove it and insert in the new position. Algorithm 4 shows this procedure.

For a standard system of fluid particles (see section 6), the number of topological events is usually small, around $10 \%$, therefore the expensive remove-insert procedure is performed only on a very few points. To improve performance the remove-insert task can be used only in the case of invalid triangulation, flipping the facets to restore the Delaunay property for the others. We note that Algorithm 4 spends a large amount of its computational time without modi- 


\section{Algorithm 4 (RECONNECTION DELAUNAY)}

(1) for every point

\{

Move the point

Compute the insphere property for the facets around it

if does not satisfy the empty sphere property

\{

Move back the point

Remove it

Insert the point in the new position

Return

\}

fying anything in the triangulation if it does not not adjust the position of the fluid particle. Because of this, the algorithm is very suited for a data-parallel parallelisation. In fact, a processor acting on a fluid particle does not invalidate the memory of any other processor, while the few topological changes can be stored in a queue and performed sequentially by each processor.

\subsection{Asynchronous time dynamics}

The physical model does not present any restrictions regarding its temporal update. In principle, but not actually implemented in the code, we can add multiscaling in time to multiscaling in space. To each fluid particle is associated a length scale given by its volume, while its time scale can be fixed by its velocity. Therefore, we can set the time scale for each fluid particle and move it on this basis. Sequential temporal updating has already been studied in particle based methods and solved with a hierarchy of time scales of power of two [20]. In practice, given the time scale for fluid particle $i, d t_{i}$, and the largest time scale $d t_{s}$, then the time steps are chosen such that

$$
d t_{i}=\frac{d t_{s}}{2_{i}^{n}}
$$

where $n_{i}$ refers to the time bin of particle $i$. Synchronisation is maintained at the end of each large time step $d t_{s}$. Then a new large time scale is computed, and so on. The Voronoi local maintenance algorithm can take advantage of this time advancement given its locality. 


\section{Simulation set-up}

In previous dissipative particle models, a priori length and time scales were fixed by the cut-off radius $[2,6,21]$. For this model, the only limiting condition is that the number of molecules $N_{k}$ in each DP must be large enough such that $1 / N_{k} \ll 1$. For the purpose of the present paper we have chosen to work with an average number of molecules per dissipative particle larger than 500 hundred. There are of course no upper bounds to the number of molecules we may assign inside a DP, but when this number is sufficiently large, the fluctuations disappear and the model becomes a standard Lagrangian hydrodynamics code. The input parameters are the size of the simulation box $L$, the number of dissipative particles $N_{D P}$, the molar mass mol, the temperature $T$ and the density $\rho$.

In our three-dimensional simulations, we set up the parameters appropriate for argon. A set of numerical values for water and argon are expressed in cgs units in Table 2. The equation of state contains the contribution of the intermolecular potential in the pairwise approximation. The size of the simulation box is set up depending on the number of DPs and the number of molecules per DP and the tessellation initialised with the Voronoi tessellation composed of regular hexagons or a random configurations of points (three dimensions) in periodic boundary conditions.

We ran a set of equilibrium simulations to benchmark the code in order to establish the computational limits of the model and the Voronoi tessellation on our machine. Because of the thermodynamic consistency of the model, the temperature obtained from the internal energy of the fluid particles is equal to the kinetic temperature, i.e. the temperature of the set of particles, computing the total kinetic energy with zero mean and converting the internal energy to the temperature. We use the set of equations (1). In three dimensions, the equilibrium simulations are performed starting from an isothermal-isobaric configuration with fixed internal energy corresponding to $300 \mathrm{~K}$ for argon but with zero momentum and equal pressures for all fluid particles. The number of fluid particles is 1000, while the number of molecules per fluid particle is set by varying the size of the system. In these simulations, we set an average of about 500 molecules per DP and a three dimensional simulation box with side $2650 \times 10^{-8} \mathrm{~cm}$. The simulations showed that the DPD temperature of the system increases until it reaches $300 \mathrm{~K}$ beyond which value the dissipative term causes the extra kinetic energy to be dissipated, operating like a thermostat. This gives strong support for the fluctuation-dissipation relations in three dimensions as well, confirming for the three-dimensional model what has been tested already in two dimensions $[9,11]$. The temperature is one of the first quantities to reach equilibrium. For momentum, mass density, pressure,

volume, etc. the equilibrium distributions can be analytically computed and 
the predictions confirmed via a more extensive series of numerical simulations which have been reported elsewhere [12].

\section{Parallel implementation and performance in two dimensions}

Whereas a parallel code can be written in two and three spatial dimensions following exactly the same strategy, we have so far implemented and tested the parallel performances of the model only in two dimensions. In fact, the parallel code uses the same Algorithm 1 to isolate boundary points as the sequential code. However, whereas in the sequential code the image points are the points in the boundary region of the opposite face, in the case of the parallel implementation they are the boundary points on an adjacent processor. The use of image points gives a straightforward parallel implementation with a message passing paradigm that in this case has been accomplished using the MPI (Message Passing Interface) [22] standard library. The big advantage of the image points solution is that this parallelisation is independent of the library used. The library is used as a software engine to compute the tessellation only.

The model contains three phases of communication:

- to construct the tessellation using the sequential code for periodic boundary conditions, but communicating the ghost region to the adjacent processor;

- to move the particles to the corresponding processor when the particles fall outside the processor's domain due to the position update;

- to preserve the force symmetry between two particles in different domains due to the stochastic force term in the Langevin equations.

The performance analysis of the code is reported for a Cray T3E (256 processors Alpha 600MHz) in Figure 8 and Table 3. All the benchmarks are run with the same configuration of 128000 fluid particles (Npart) for 50 iterations.

The simulation is a relaxation towards equilibrium of the isothermal model [9] starting from a random initial configuration. The timings have been taken measuring the CPU-clock time for the routines: move updates the position and communicates the particle outside the boundary, construct constructs the tessellation, integration integrates the Langevin equations and communicates the stochastic force, comm is the total time of communication without the time for buffering and total measures the total time without Input-Output. All the times measured are inclusive of communication, which is reported separately for comparison; the time for one processor is the time of the sequential version without any overhead due to buffering. 
The important point which we wish to stress here is that although the construction and maintenance of the Voronoi tessellation represents a significant computational overhead due to the algorithmic complexity of $N \log N$, with this parallel implementation the speed-up obtainable is very promising. This is an intrinsic feature of the model. Each fluid particle interacts only with its nearest neighbour, thereby reducing the amount of communication. Previous standard dissipative particle methods [6,23] lacked this strong locality feature, interacting with many more neighbouring particles and severely limiting the parallel performance $[24,25]$.

Looking at the speed-up index, it is worth noting that the super-linearity shown was expected because the algorithmic complexity of the construction is not linear, but $N \log (N)$. The construction of the tessellation for $N / 2$ takes less than half of the time spent for $N$ dissipative particles.

The present parallel implementation may suffer from load imbalance depending on the nature of the multiscaling in a given application. If the length scale is set by the polymer molecule and the polymer density is uniform in the simulation region then the parallel implementation should be well balanced. A different situation may arise in the case of multiscaling due to the flow structure, when some processors may have a higher computational load than others. In this case the parallel performance will drop to the slowest processor's performance. A dynamic repartitioning of the simulation region among processors should reduce this effect, but the repartitioning routine would necessitate further communication, thereby producing an additional overhead.

\section{Computational limits}

In the preceding sections, we described several algorithms that we have used to implement boundary conditions and dynamic reconstruction of the tessellation. We showed as well the good parallel performance of the model due to the locality of its interactions. Now we want to estimate the computational limits of this model in view of future applications to complex fluids flows. The CGAL library version 2.3 is used for these runs.

In two dimensions, a sequential code can easily handle 50,000 fluid particles. Considering the good parallel performance, it is possible to run very large scale simulations. The situation in three dimensions is quite different. The poor efficiency of periodic boundary conditions limits the overall performance of the code. In Table 4, we report the computational time in seconds for a set of runs with increasing number of fluid particles, for 10 iterations. The simulation is a simple equilibration of the fluid particle system. We also report the average number of topological events (reconnections) that are performed for each 
iteration, the time to move and maintain all the fluid particles, the Eulerian time corresponding to the time needed to solve the Langevin equations once the tessellation and the volumes have been computed, and finally the time to impose boundary conditions.

Table 4 suggests that while the maintenance of the tessellation is reasonable compared to the time needed to integrate the Langevin equations, the boundary conditions do not allow to increase the size of the simulation to more than few thousands fluid particles. For the maintenance task, a non-local reconnection algorithm, which restores the Delaunay property after the entire set of points are moved, would be at least 3 times faster in the move task. However, the stability would be affected and the possibility of asynchronous time update is also lost. Instead, our code proved to be very stable. Further optimisations are of course possible using local flips and remove-insert procedures only in pathological configurations.

\section{Conclusions}

Within the framework of fluctuating hydrodynamics codes for complex fluids, we have described our present algorithmic approach to multiscale dissipative particle dynamics. The algorithms to dynamically maintain the Voronoi tessellation, impose boundary conditions and develop parallel codes for the Voronoi dissipative particle dynamics model have been described in two and three spatial dimensions. The use of publicly available computational libraries $[16,17]$ is an advantage compared to direct coding because the evolution of the library is automatically incorporated into the model. This is an important factor at this stage of research in computational geometry where a really stable and efficient library is still under development. In this context, CGAL offers a first interesting approach, delivering a usable and powerful tool for scientists.

The reconnection algorithm used to dynamically maintain the tessellation in two dimensions is stable as long as it is applied on a valid albeit not Delaunay triangulation. This is not easy to guarantee even reducing the time step. In three dimensions, our approach in Section 5.2 has a limited additional cost, but offers the advantage of great stability. The overall efficiency is good for running simulations in real applications.

Boundary conditions are evidently not yet efficiently addressed in three dimensions. Periodic boundaries take most of the time in the simulation. The image points approach for boundaries is appealing because it provides at the same time periodic, fixed wall boundary and a domain decomposition parallel implementation, but it does not perform efficiently due to the large number of image points and the complexity and cost of the point removal from the tes- 
sellation in three dimensions. However, it is possible to use periodic boundary in the $x$ direction and a wall surface boundary on the $y$ and $z$ to significantly reduce the amount of image points. This corresponds to flowing a fluid in a duct, where shear flows can be applied to compute the rheological properties of the fluid.

Alternative implementations of three dimensional periodic boundary conditions can be envisaged. Although, we have not actually implemented it, it is nevertheless interesting to consider here an alternative option. Imposing periodic boundary conditions on a bounded region of space can be thought as actually bending the Euclidian space of the region to form a torus. This is actually equivalent to changing the topology of the region which, in fact, might lead to problems of finite size effects (in case the triangulation becomes locally non-euclidian). We have implemented boundary conditions by imposing periodicity on the Euclidian space around a box of fixed size. This makes use of image points outside the box in order to obtain the periodic boundary. A second alternative is to use a three dimensional torus, without image points, but directly connecting the fluid particles from one side to the other. Of course, this necessitates some care when handling the coordinates. A possible implementation of this solution requires changing the insphere function which is used to construct the tessellation. This is related to the distance function, which could be implemented as $d_{\text {torus }}\left(\mathbf{P}_{1}, \mathbf{P}_{2}\right):=\operatorname{norm}\left(\left(\mathbf{P}_{1}-\mathbf{P}_{2}\right) \bmod (\mathbf{L} / 2)\right)$, where $\mathbf{L}=\left\{L_{x}, L_{y}, L_{z}\right\}$ represents the three linear dimensions of the box and the modulus operation mod is applied to each component. This function must consider the toroidal topology, in order that points close to the boundary of the previous Euclidian box are still considered close as they are in the torus. This implementation would require substantial modifications of the CGAL library.

\section{Acknowledgements}

G.D.F. thanks Queen Mary, University of London, and Schlumberger Cambridge Research for funding his Ph.D studentship. We thank CGAL for help and support, and P. Español and M. Serrano for useful discussions and exchange of preprints.

\section{A Maintaining the Voronoi tessellation}

In two dimensions, we are able to restore the Delaunay triangulation after every time-step with the algorithm listed below, which is an extension of the restore_delaunay function in Delaunay_triangulation_2.h. We first compute the 
time-step such that no Delaunay vertex can move outside its Voronoi region. This helps to ensure that we have a valid triangulation, but it does not guarantee it. We integrate the stochastic Langevin equations of motion and then update the position of each vertex by calling the CGAL set_point function. For each vertex $v$, we then circulate around the incident faces, checking that $v$ is not located inside the circumcircle of each neighbour of $v$ (external flip) and that no vertices adjacent to $v$ lie inside the circumcircle of each face (internal flip). For any invalid Delaunay triangle, we simply triangulate the quadrilateral the other way by calling the CGAL flip function. Thus we are able to efficiently restore the two dimensional Voronoi tessellation locally.

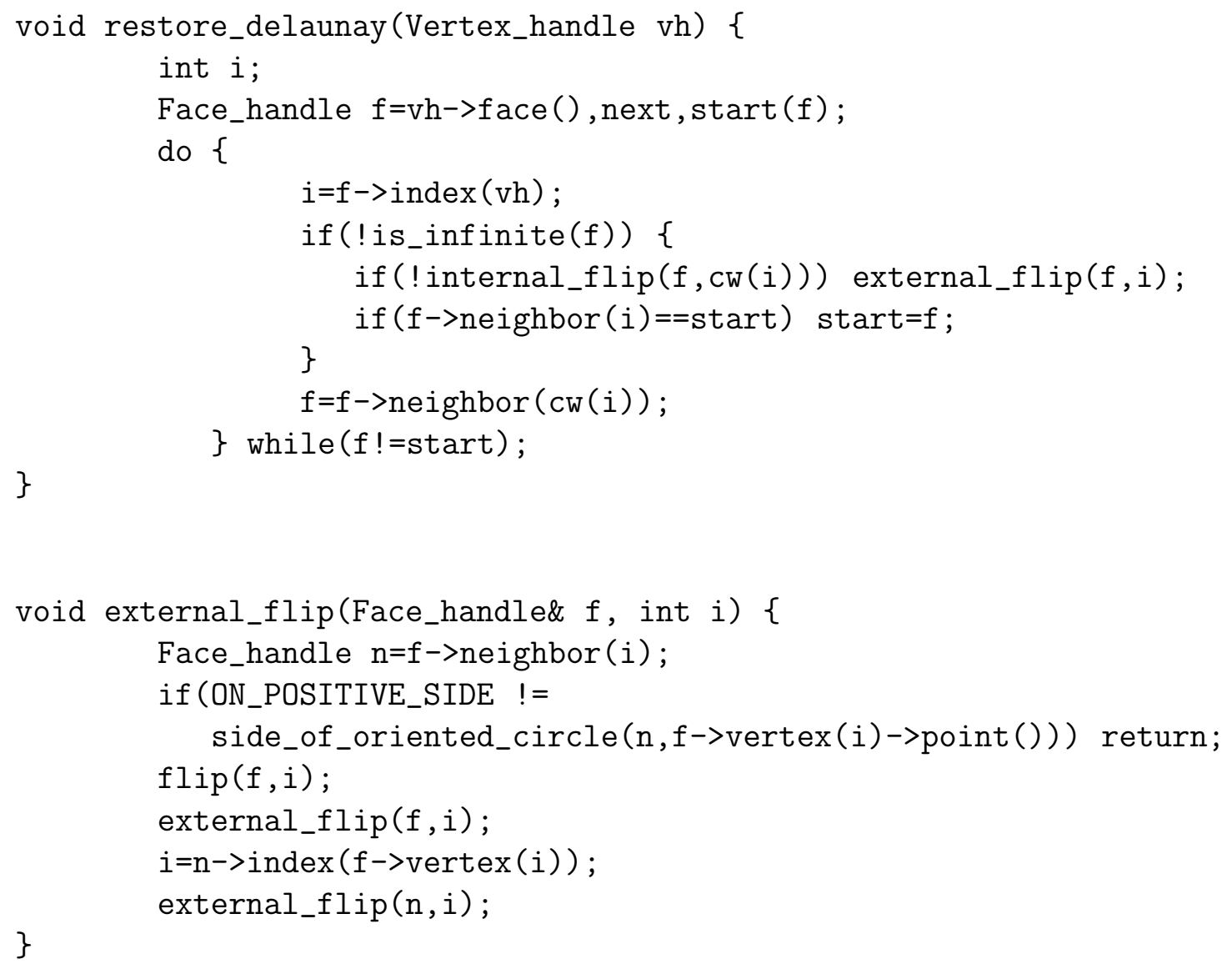


In three dimensions, the in_sphere property is checked for all the facets incidents to a given vertex vh. If a topological event is found then the point is removed and inserted again. The facets are inserted in a set in order to avoid double checking the same facet. An implementation is listed below.

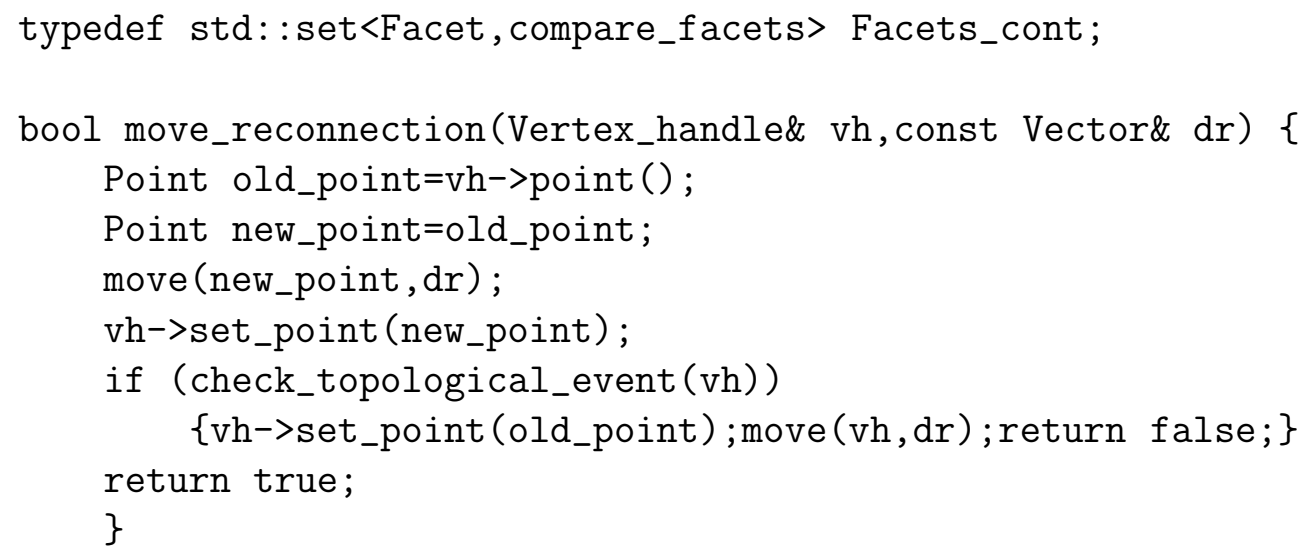




\section{References}

[1] X.-F. Yuan and M. Doi, Colloids and surfaces A 144, 305 (1998).

[2] P. J. Hoogergrugge and J. M. V. A. Koelman, Europhys. Lett. 19, 155 (1992).

[3] E. S. Boek, P. V. Coveney, H. N. W. Lekkerkerker, and P. van der Schoot, Phys. Rev. E 54, 5143 (1997).

[4] A. G. Schlijper, P. J. Hoogerbrugge, and C. W. Manke, J. Rheol. 39, 567 (1995).

[5] P. V. Coveney and K. E. Novik, Phys. Rev. E 54, 5143 (1996).

[6] P. Español and P. Warren, Europhys. Lett. 30, 191 (1995).

[7] C. A. Marsh, G. Backx, and M. Ernst, Phys. Rev. E 56, 1676 (1997).

[8] E. G. Flekkøy and P. V. Coveney, Phys. Rev. Lett. 83, 1775 (1999).

[9] E. G. Flekkøy, P. V. Coveney, and G. De Fabritiis, Phys. Rev. E 62, 2140 (2000).

[10] G. De Fabritiis, P. V. Coveney, and E. G. Flekkøy, Phil. Trans. Royal Soc. Lond. A 360, 317 (2002).

[11] M. Serrano and P. Español, Phys. Rev. E 64, 046115 (2001).

[12] M. Serrano, G. De Fabritiis, P. Español, and P. V. Coveney, J. Phys. A: Math. Gen. 35, 1605 (2002).

[13] P. E. Kloeden and E. Platen, Numerical solution of stochastic differential equations, Springer-Verlag, Berlin, 1992.

[14] G. De Fabritiis, M. Serrano, P. Español, and P. V. Coveney, Efficient integrators for mesoscopic models based on stochastic differential equations, preprint, 2002.

[15] J. Rourke, Computational geometry in C, Cambridge University Press, Cambridge, 1994.

[16] J. R. Shewchuk, (www.cs.cmu.edu/ quake/triangle.html).

[17] Computational Geometry Algorithms Library (www.cgal.org).

[18] G. De Fabritiis, P. V. Coveney, and E. G. Flekkøy, Proceedings of the 5th European SGI/Cray MPP Workshop, Bologna, Italy (1999).

[19] G. Albers, L. J. Guibas, J. S. B. Mitchel, and T. Ross, Int. J. of Comp. Geom. \& App. 8, 365 (1998). 
[20] L. Hernquist and N. Kats, Astrophys. J. Suppl. Series 70, 419 (1989).

[21] J. B. Avalos and A. D. Mackie, Europhys. Lett. 40, 141 (1997).

[22] MPI: A message-passing interface standard (www.mpi-forum.org).

[23] J. J. Monaghan, Ann. Rev. Astron. Astrophys. 30, 543 (1992).

[24] S. I. Jury, P. Bladon, S. Krishna, and M. E. Cates, Phys. Rev. E 59, R2535 (1999).

[25] K. E. Novik and P. V. Coveney, Phys. Rev. E 61, 435 (2000). 
Table 1

Number of image points inserted in two and three spatial dimensions to impose boundary conditions.

\begin{tabular}{ccccc}
\hline No. of DPs & 2D images & 3D shell & 3D images & 3D extra images \\
\hline 100 & 64 & 100 & 408 & 195 \\
1000 & 190 & 687 & 1329 & 398 \\
10000 & 581 & 3855 & 5287 & 661 \\
50000 & 2081 & 11998 & 14370 & 1050 \\
\hline
\end{tabular}

Table 2

The input parameters for argon and water used for the simulations. All the numerical values are expressed in cgs units for three spatial dimensions. $n=N / N_{A}, N_{A}$ is Avogadro's number and $E_{i d}$ is the internal energy of an ideal gas.

\begin{tabular}{lcc}
\hline cgs units & argon & water \\
\hline temperature & 300 & 300 \\
viscosity & $2.26 \mathrm{E}-4$ & 0.01 \\
heat diffusivity & $2 \mathrm{E} 3$ & $5.9 \mathrm{E} 4$ \\
mass density & 0.00178 & 1 \\
molecular mass & 39.94 & 18 \\
pressure equation of state & $P V=N k_{B} T$ & $\left(P+\frac{n^{2} a}{V^{2}}\right)(V-n b)=N k_{B} T$ \\
\hline
\end{tabular}


Table 3

Timings in seconds of the main routines on a Cray T3E. Comm measures the total time for communication, while the other timings are inclusive of the communication time. The number of iterations is 50 .

\begin{tabular}{ccccccc}
\hline Nprocs & Npart/NProcs & Move & Construct & Integration & Comm & Total \\
\hline 1 & 128000 & 4.50 & 395.17 & 19.38 & 0 & 427.73 \\
2 & 64000 & 5.45 & 180.96 & 16.54 & 1.49 & 206.99 \\
4 & 32000 & 2.76 & 83.42 & 8.46 & 1.43 & 96.52 \\
8 & 16000 & 1.48 & 38.48 & 4.43 & 1.02 & 45.34 \\
16 & 8000 & 0.78 & 17.70 & 2.24 & 0.67 & 21.24 \\
32 & 4000 & 0.44 & 8.40 & 1.16 & 0.44 & 10.31 \\
64 & 2000 & 0.25 & 4.09 & 0.68 & 0.38 & 5.18 \\
128 & 1000 & 0.17 & 2.14 & 0.49 & 0.47 & 2.91 \\
\hline
\end{tabular}

Table 4

Computational time in seconds for 10 iterations of the three dimensional tessellations with periodic boundary conditions.

\begin{tabular}{lcccc}
\hline Fluid particles & Reconnected & Move & Eulerian & Update images \\
\hline 100 & 5 & $0.86 \mathrm{~s}$ & $0.87 \mathrm{~s}$ & $17.5 \mathrm{~s}$ \\
1000 & 70 & $9.5 \mathrm{~s}$ & $1.0 \mathrm{~s}$ & $65.6 \mathrm{~s}$ \\
5000 & 450 & $49.0 \mathrm{~s}$ & $5.0 \mathrm{~s}$ & $201.5 \mathrm{~s}$ \\
\hline
\end{tabular}




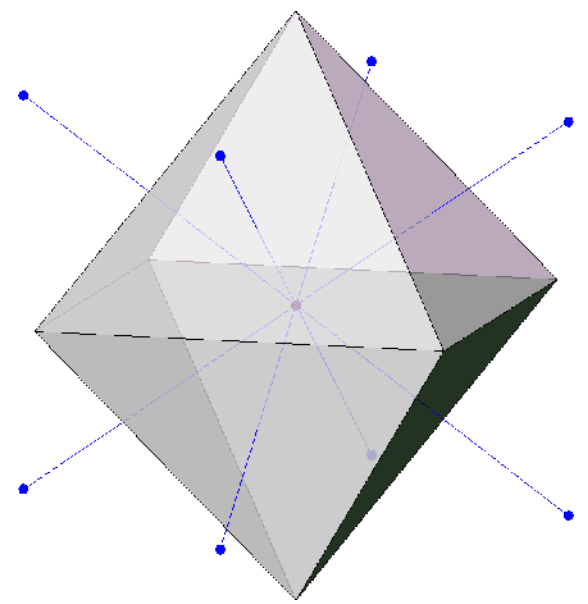

Fig. 1. A fluid particle in three dimensions for the simple case of a regular grid. The stresses and fluxes are computed over the surfaces between the Voronoi centre points.

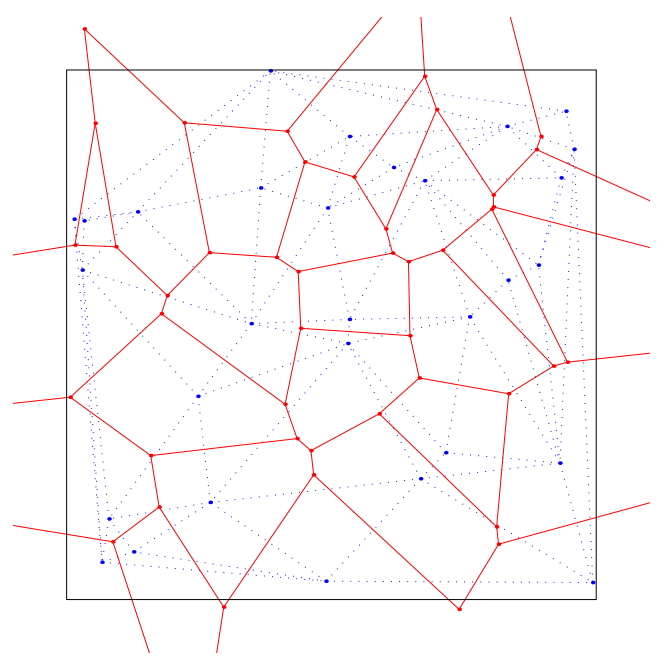

Fig. 2. The tessellation for an unbounded domain. The continuous lines are the Voronoi edges, while the points represent the dissipative particles or Delaunay vertices. The dotted lines are the edges of the Delaunay triangulation and the missing Voronoi edges are infinite edges. The square is the simulation domain. 


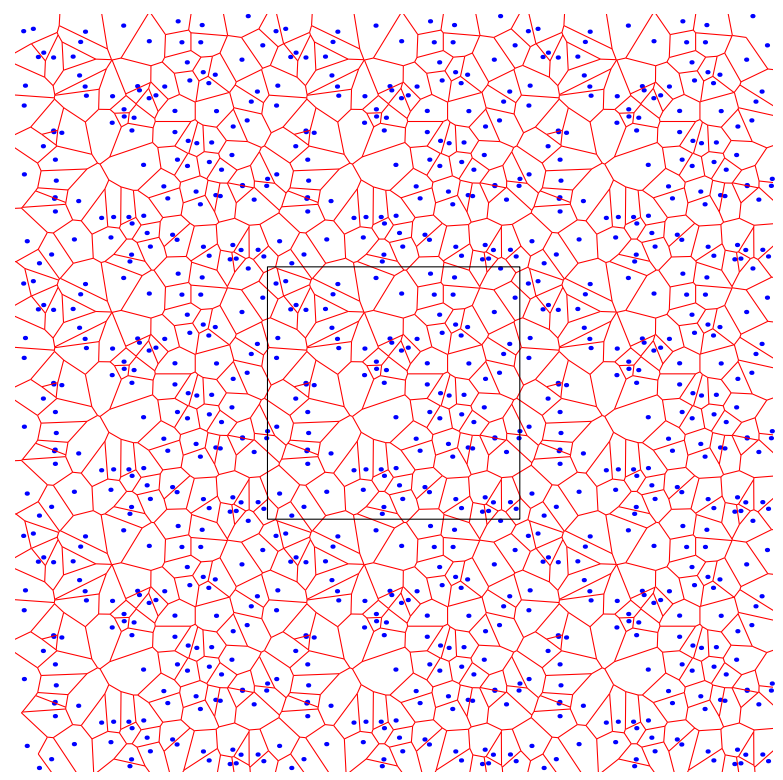

Fig. 3. The simplest way to construct a periodic tessellation. The points in the simulation region are copied to the corresponding positions in the eight surrounding boundary regions to produce a periodic Voronoi tessellation.

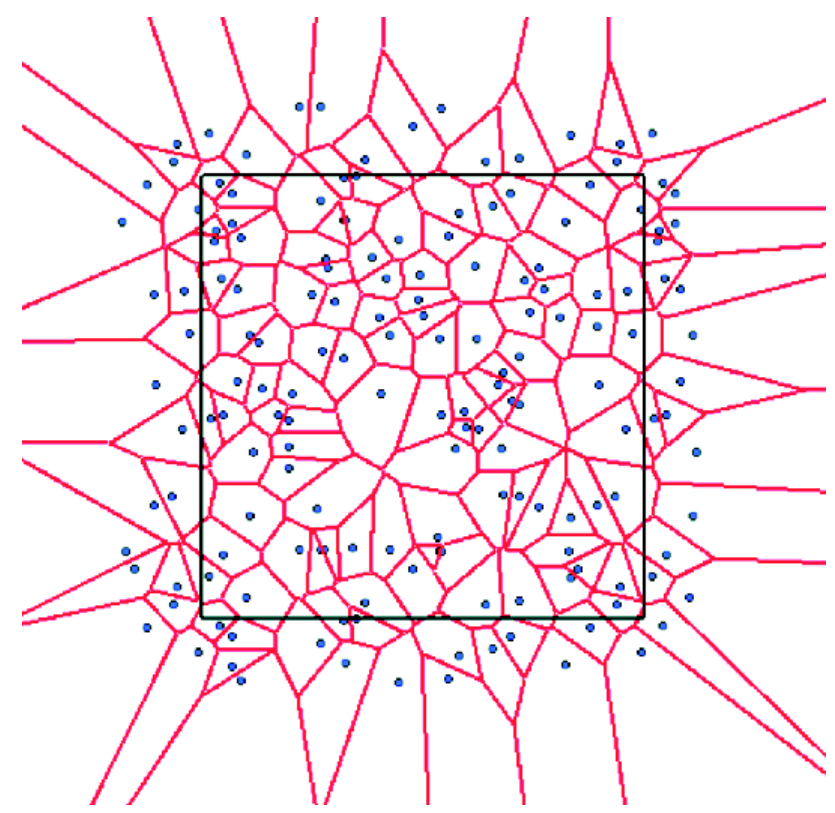

Fig. 4. Implementation of periodic boundary conditions using Algorithm 1. 


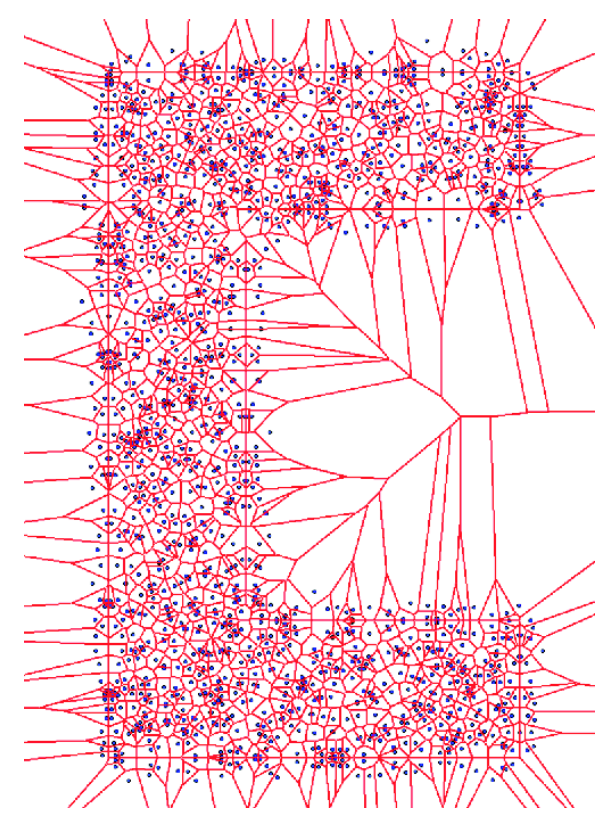

Fig. 5. Implementation of fixed (solid) wall boundary conditions using Algorithm 2.

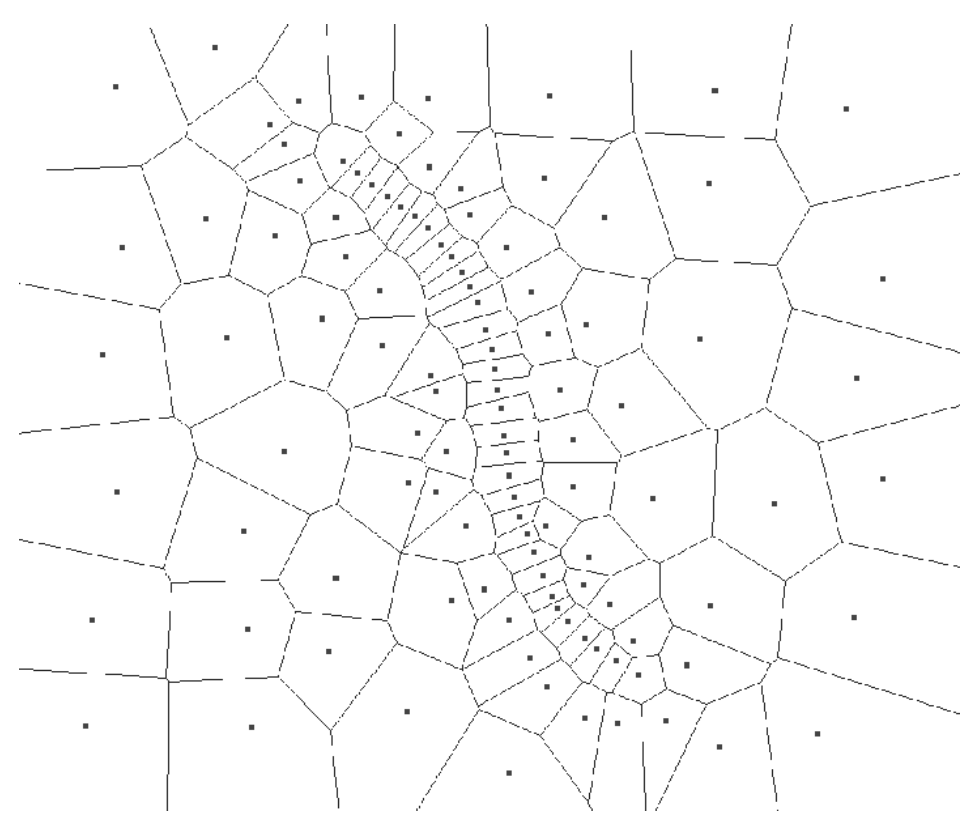

Fig. 6. Structure of a polymeric fluid according to the multiscale dissipative particle dynamics method. The resolved length scale of the polymer is much finer than that of the fluid. In the fluid itself different length scales are present to handle polymer-fluid interactions. 


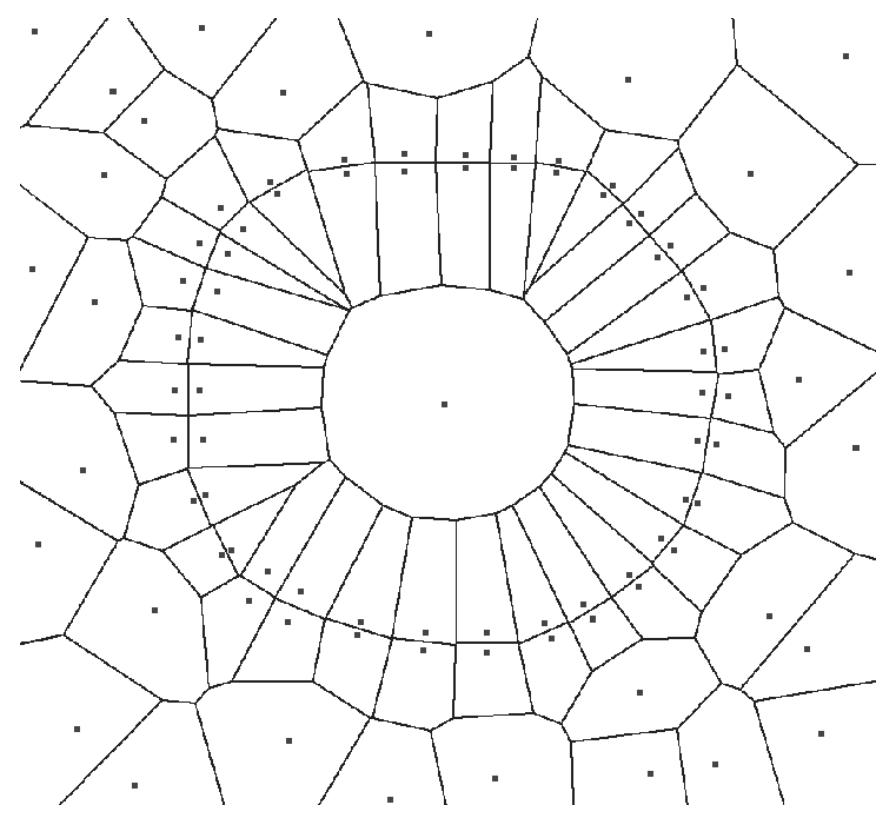

Fig. 7. Structure of a colloidal particle according to the multiscale dissipative particle dynamics method. The surface of the colloid is resolved by inserting two points for each surface element. The central point represents the colloid's centre of mass and is used to make the tessellation more stable.

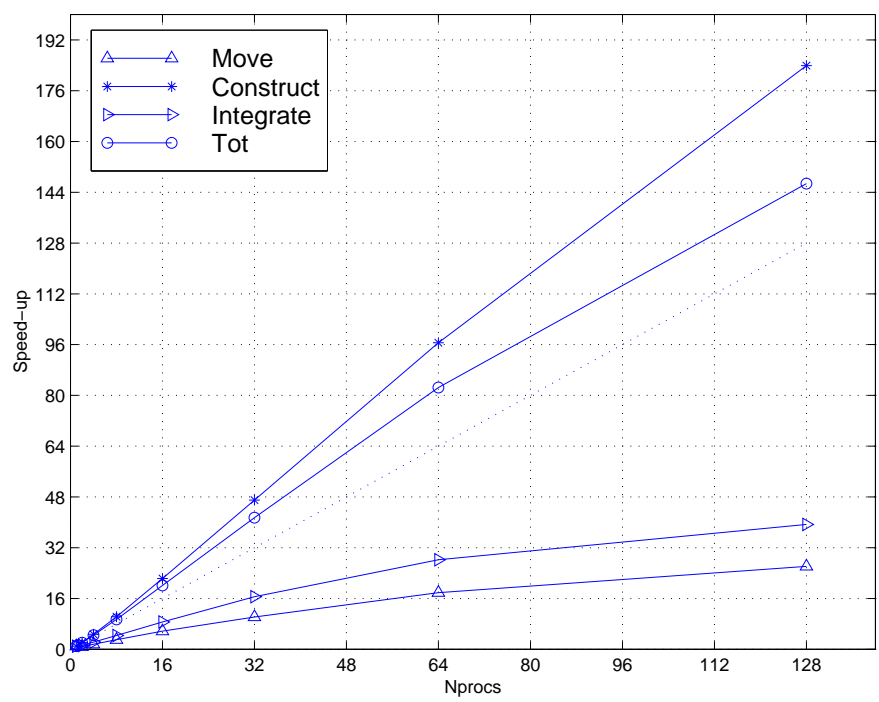

Fig. 8. Speed-up index on a Cray T3E. The dotted line indicates the linear speed-up. Circles indicate overall speed-up, stars Voronoi construction speed-up, triangles the speed-up of the integration and update of the Langevin equations. 